\title{
Ultrafast Dynamics of Fluctuations in High-Temperature Superconductors Far from Equilibrium
}

\author{
L. Perfetti, ${ }^{1}$ B. Sciolla, ${ }^{2}$ G. Biroli, ${ }^{3}$ C. J. van der Beek, ${ }^{1}$ C. Piovera, ${ }^{1}$ M. Wolf, ${ }^{4}$ and T. Kampfrath ${ }^{4}$ \\ ${ }^{1}$ Laboratoire des Solides Irradiés, Ecole polytechnique, 91128 Palaiseau cedex, France \\ ${ }^{2}$ Departement of Theoretical Physics, Ecole de Physique University of Geneva 24, Quai Ernest Ansermet 1211 Genéve, Switzerland \\ ${ }^{3}$ Institut de Physique Théorique CEA, (CNRS URA 2306), 91191 Gif-sur-Yvette, France \\ ${ }^{4}$ Fritz Haber Institute of the Max Planck Society, Faradayweg 4-6, 14195 Berlin, Germany
}

(Received 14 September 2014; revised manuscript received 23 December 2014; published 12 February 2015)

\begin{abstract}
Despite extensive work on high-temperature superconductors, the critical behavior of an incipient condensate has so far been studied exclusively under equilibrium conditions. Here, we excite $\mathrm{Bi}_{2} \mathrm{Sr}_{2} \mathrm{CaCu}_{2} \mathrm{O}_{8+\delta}$ with a femtosecond laser pulse and monitor the subsequent nonequilibrium dynamics of the midinfrared conductivity. Our data allow us to discriminate temperature regimes where superconductivity is either coherent, fluctuating or vanishingly small. Above the transition temperature $T_{\mathrm{c}}$, we make the striking observation that the relaxation to equilibrium exhibits power-law dynamics and scaling behavior, both for optimally and underdoped superconductors. Our findings can in part be modeled using time-dependent Ginzburg-Landau theory, and they provide strong indication of universality in systems far from equilibrium.
\end{abstract}

DOI: 10.1103/PhysRevLett.114.067003

Many physical properties of high-temperature superconductors cannot be understood in the framework of a mean-field theory. For example, an incipient condensate with short coherence length fluctuates in space and time, leading to precursor effects of the superconducting phase. As a consequence, quantities such as conductivity [1-4], heat capacity [5], diamagnetic susceptibility [6], and the Nernst signal [7] may increase considerably when the temperature approaches the critical value $T_{\mathrm{c}}$. For cuprates, most of these observations have successfully been described by the Ginzburg-Landau (GL) model or quantum-field theories [8-11]. Suitable extensions of these concepts to systems in nonequilibrium states predict critical dynamics and scaling laws [12]. Critical exponents independent of specific material properties may help extend the idea of "universality classes" [13] to systems far from equilibrium. These phenomena should not only occur in condensedmatter systems, but also in high-energy physics and ultracold atomic gases [12,14].

An ideal protocol for the detection of critical dynamics requires that superconducting correlations are monitored just above the transition temperature, where the system is expected to exhibit universal behavior. To induce and probe the nonequilibrium regime, ultrafast pump-probe spectroscopy is a very promising approach [15-26]. Unfortunately, in experiments using optical probes (photon energy of typically $1.5 \mathrm{eV}$ ), it is not straightforward to detect superconducting fluctuations above $T_{\mathrm{c}}$ because of the presence of competing signals ascribed to the pseudogap [15,17]. To disentangle fluctuation- and pseudogap-related signal components, an optical three-pulse technique was proposed recently [19]. Alternatively, electromagnetic pulses covering the frequency range from about 1 to $3 \mathrm{THz}$ have been shown to be highly sensitive probes of the superconducting condensate [22-25]. However, the duration of the $\mathrm{THz}$
PACS numbers: 74.40.Gh, 74.25.Gz, 74.72.Gh, 78.30.Er

pulses $(\sim 0.5 \mathrm{ps})$ is too long to resolve the ultrafast dynamics of the superconductor.

In this Letter, we monitor superconducting fluctuations by time-resolved detection of the conductivity in the midinfrared (MIR). Thin films of the superconductor $\mathrm{Bi}_{2} \mathrm{Sr}_{2} \mathrm{CaCu}_{2} \mathrm{O}_{8+\delta}$ (BSCCO) are excited by optical laser pulses and probed by electromagnetic pulses with a center frequency of $20 \mathrm{THz}$ and a duration of $\sim 100 \mathrm{fs}$. The corresponding photon energy of $80 \mathrm{meV}$ is comparable to the single-particle gap of BSCCO, thereby making MIR pulses a highly sensitive yet still ultrafast probe of superconducting correlations $[20,21]$. By these means, we identify a critical scaling behavior and a universal power law of the ultrafast nonequilibrium dynamics of the incipient condensate, both for optimally and underdoped samples. Part of our data can be reproduced by the timedependent Ginzburg-Landau theory, a coarse-grained model justified near to the critical temperature. Our results suggest a promising route to study the highly unexplored field of critical behavior far from equilibrium.

Experimental details.-Samples used are 150-nm thin films of optimally doped $\left(T_{\mathrm{c}}=91 \mathrm{~K}\right)$ and underdoped $\left(T_{\mathrm{c}}=68 \mathrm{~K}\right) \mathrm{BSCCO}$ mounted on a diamond substrate that is transparent to both pump and probe radiation. The MIR spectrometer is driven by laser pulses from a Ti:sapphire laser oscillator (pulse duration of $10 \mathrm{fs}$, center wavelength of $780 \mathrm{~nm}$, repetition rate of $80 \mathrm{MHz}$ ). Part of the laser output is used to generate and detect MIR pulses [21] ( $\sim 100 \mathrm{fs}$, spectrum from 10 to $30 \mathrm{THz}$ ), whereas the remainder is used to excite the sample with an incident fluence of about $16 \mu \mathrm{J} \mathrm{cm}^{-2}$. We simultaneously measure the MIR electric fields $E_{0}(t, T)$ and $E(t, \tau)$ where $t$ denotes time with respect to the field maximum at $t=0$ [see Fig. 1(a)], and $\tau$ is the delay between pump and probe pulse. 


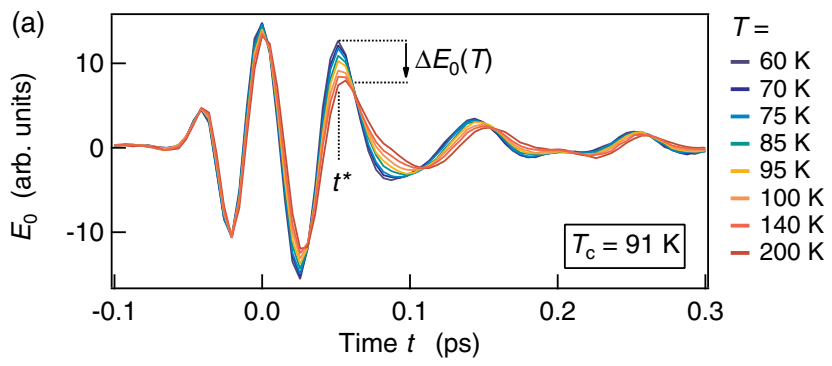

(b) Energy (meV)
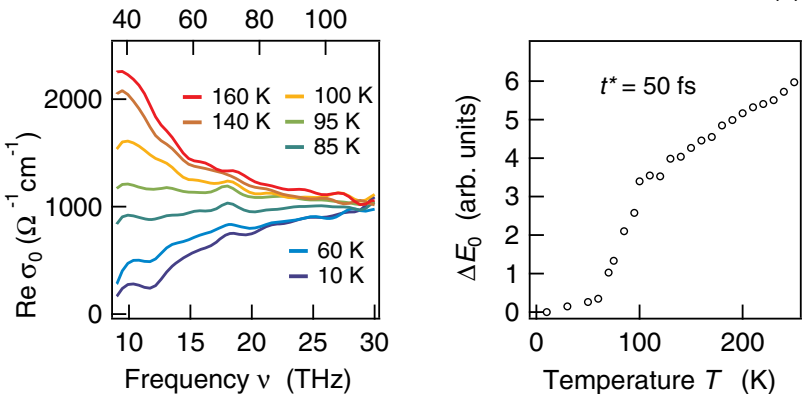

FIG. 1 (color online). Equilibrium data of optimally doped BSCCO $\left(T_{\mathrm{c}}=91 \mathrm{~K}\right)$. (a) Electric field $E_{0}(t, T)$ of the MIR waveform transmitted through the BSCCO film as a function of time $t$. Each sample temperature $T$ is indicated by a different line color. (b) Real part $\operatorname{Re} \sigma_{0}(\nu)$ of the MIR conductivity versus frequency $\nu$ and (c) change $\Delta E_{0}(T)=E_{0}\left(t^{*}, 6 \mathrm{~K}\right)-E_{0}\left(t^{*}, T\right)$ in the transmitted MIR field at time $t^{*}=50 \mathrm{fs}$ as a function of $T$.

While $E_{0}(t, T)$ quantifies the field transmitted through the sample in thermodynamic equilibrium at temperature $T$, $E(t, \tau)$ is the field in the case of the pumped sample.

Equilibrium case.-Figure 1(a) shows MIR field traces $E_{0}(t, T)$ after having traversed optimally doped BSCCO films in equilibrium at various temperatures $T$. To extract the conductivity $\sigma(\nu)$ as a function of MIR frequency $\nu$, we apply a Fourier transformation and standard Fresnel formulas to the data of Fig. 1(a). The real part of the MIR conductivity is shown in Fig. 1(b), and we find that $\operatorname{Re} \sigma(\nu)$ decreases with frequency for $T>T_{\mathrm{c}}=91 \mathrm{~K}$, whereas it follows the opposite trend in the superconducting phase with $T<T_{\mathrm{c}}$. These results are in excellent agreement with previous reports based on Fourier transform spectroscopy [27] and ellipsometry [28,29].

A detailed analysis of the probing process [30] shows that the variation $\Delta E_{0}(T)=E_{0}\left(t^{*}, 6 \mathrm{~K}\right)-E_{0}\left(t^{*}, T\right)$ of the MIR electric field at the maximum position $t^{*}=50 \mathrm{fs}$ is proportional to the real part of the difference $\sigma_{0}(\nu, 6 \mathrm{~K})-$ $\sigma_{0}(\nu, T)$ averaged over the frequency interval from 15 to $25 \mathrm{THz}$. Figure 1(c) displays the temperature dependence of $\Delta E_{0}(T)$. When temperature is lowered from 300 to $100 \mathrm{~K}$, we observe a linear decrease, that turns into an abrupt drop at the transition to the superconducting phase $\left(T \sim T_{\mathrm{c}}=91 \mathrm{~K}\right)$. The physical interpretation of this sudden decrease is straightforward: the emergence of the superconductivity gap $\left(T \sim T_{\mathrm{c}}\right)$ reduces the density of electronic states (DOS) in the vicinity of the Fermi energy, thereby reducing the number of scattering channels of the electrons [31]. As a consequence, there is much less dissipation of the driving MIR electric field below $T_{\mathrm{c}}$.

In the fluctuating regime above $T_{\mathrm{c}}$, the DOS becomes a function of local gap amplitude and of the superconducting coherence length $\xi$ [31]. The latter reflects the typical distance over which the superconducting order parameter maintains its amplitude and phase [8], and it is expected to diverge exactly at the transition temperature. Theoretical models of two-dimensional superconductors [32] predict that the MIR conductivity scales with $\ln \left(\xi / \xi_{0}\right)$ where $\xi_{0}$ is the value of the coherence length at zero temperature. Therefore, MIR pulses are an excellent probe of superconducting fluctuations [3].

Nonequilibrium case.-We now photoexcite the BSCCO sample with an optical pump pulse (duration of $10 \mathrm{fs}$, photon energy of $1.55 \mathrm{eV}$ ) and monitor the resulting ultrafast dynamics. According to previous work [25,33], our pump fluence of $16 \mu \mathrm{J} \mathrm{cm}^{-2}$ is large enough to reduce the order parameter by a fraction on the order of $80 \%$. Figure 2(a) shows the pump-induced change $\Delta E(\tau)=E\left(t^{*},-1 \mathrm{ps}\right)-E\left(t^{*}, \tau\right)$
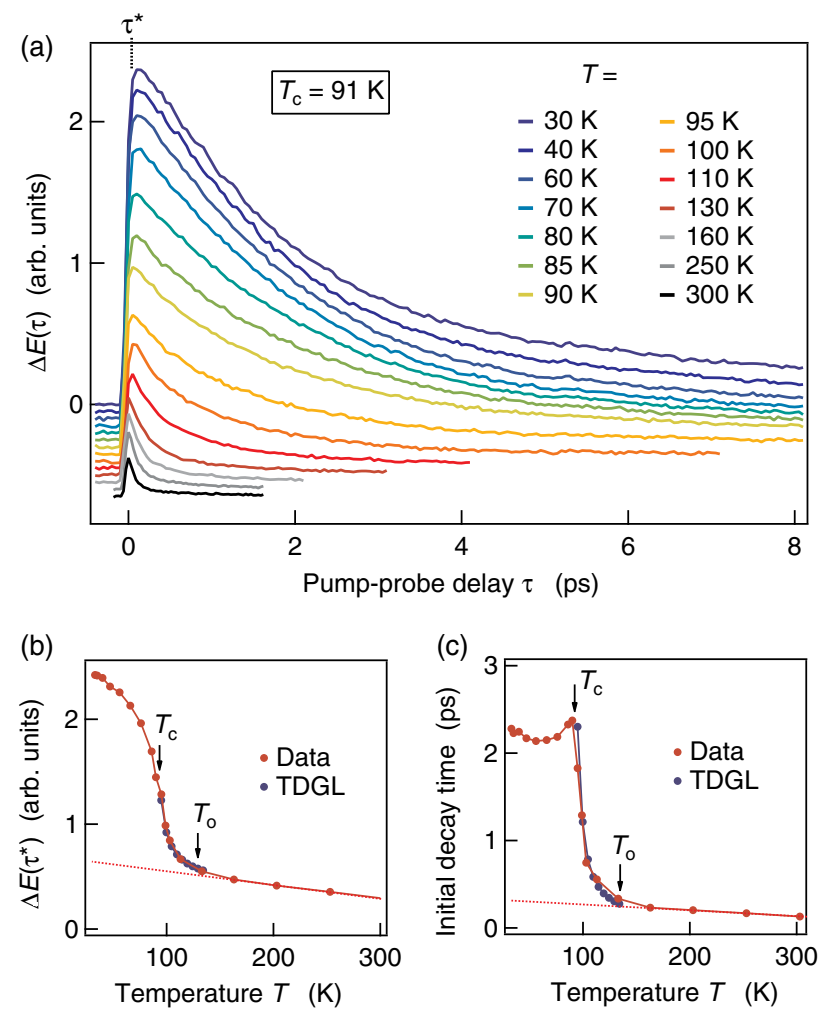

FIG. 2 (color online). Nonequilibrium data of optimally doped BSCCO $\left(T_{\mathrm{c}}=91 \mathrm{~K}\right)$. (a) Pump-induced changes $\Delta E(\tau)=$ $E\left(t^{*},-1 \mathrm{ps}\right)-E\left(t^{*}, \tau\right)$ in the transmitted MIR electric field as a function of pump-probe delay $\tau$. $\Delta E(\tau)$ scales with the pumpinduced change $\operatorname{Re} \Delta \sigma(\nu, T)$ in the instantaneous BSCCO conductivity averaged from 15 to $25 \mathrm{THz}$. (b) Signal magnitude $\Delta E$ and (c) relaxation time $(\partial \ln \Delta E / \partial \tau)^{-1}$ directly after sample excitation ( $\tau=\tau^{*}=50 \mathrm{fs}$ ) as a function of temperature (red circles). Blue squares result from a model based on the TDGL equation. The transition temperature $\left(T_{\mathrm{c}}=91 \mathrm{~K}\right)$ and the onset of superconducting fluctuations (at $T_{0}=130 \mathrm{~K}$ ) are indicated by arrows. 
in the transmitted MIR field for the optimally doped sample as a function of the pump-probe delay $\tau$ at various ambient temperatures $T$. As above, we consider the MIR fields at time $t^{*}=50$ fs such that $\Delta E(\tau)$ scales with the pump-induced change $\operatorname{Re} \Delta \sigma(\nu, \tau)$ in the instantaneous BSCCO conductivity averaged from 15 to $25 \mathrm{THz}$ [30]. As shown by Fig. 2(a), magnitude and recovery time of $\Delta E(\tau)$ strongly depend on sample temperature. Also, the dynamics deviates substantially from an exponential decay and slows down with increasing pump-probe delay.

To better illustrate the temperature dependence of the curves in Fig. 2(a), we extract two characteristic parameters, namely the signal magnitude $\Delta E(\tau)$ and the instantaneous decay time $(\partial \ln \Delta E / \partial \tau)^{-1}$ directly after sample excitation at $\tau=\tau^{*}=50 \mathrm{fs}$. The latter is obtained by fitting the dynamics with an exponential fit in a small time interval just after photoexcitation. The two parameters are displayed as a function of sample temperature $T$ in Figs. 2(b) and 2(c). As seen in Fig. 2(b), $\Delta E\left(\tau^{*}\right)$ is much larger when $T$ is below the transition temperature. Above $T_{\mathrm{c}}$, we still observe an appreciable $\Delta E\left(\tau^{*}\right)$ that, above a temperature $T_{0}$, turns into a relatively flat and linearly decreasing curve (see arrows). Figure 2(c) shows that the initial decay time of the pump-probe signal exhibits an analogous behavior: it is large below $T_{\mathrm{c}}$, then drops sharply and turns into a flat curve above $T_{0}$. We attribute these temperature intervals to the three different regimes: a superconducting phase $\left(T<T_{\mathrm{c}}\right)$, a fluctuating superconductor $\left(T_{\mathrm{c}}<T<T_{0}\right)$, and a metallic phase $\left(T>T_{0}\right)$. From Fig. 2, we obtain the rough estimate $T_{0}=130 \mathrm{~K}$, which is in good agreement with values extracted from measurements of transport [1], specific heat [5], diamagnetic susceptibility [6], and the Nernst effect [7].

Ultrafast scaling behavior.-In the temperature region from $T=T_{0}$ down to the critical temperature $T_{\mathrm{c}}$, the system is governed by an increasing coherence length $\xi$ [8]. The characteristic time $\tau_{\mathrm{c}}$ it takes a Cooper pair to cover the coherence length should increase accordingly. The scaling hypothesis suggests that the relaxation should follow a universal power law $\left[\tau / \tau_{\mathrm{c}}(T)\right]^{-\alpha}$ where $\alpha$ is a critical exponent that only depends on the observable considered, the symmetry of the order parameter, and the dimension of the system [34].

Figure 3 shows the key result of this Letter: the striking observation that the apparently diverse dynamics of Fig. 2(a), indeed, obey a scaling law. As seen in Fig. 3, all decay curves for $T_{\mathrm{c}}<T<T_{0}$ fall onto one when the time axis $\tau$ is normalized by a suitable time $\tau_{\mathrm{c}}(T)$. More precisely, we find that the relation

$$
\Delta E(\tau)=\frac{\Delta E\left(\tau^{*}\right)}{1+\left[\tau / \tau_{\mathrm{c}}(T)\right]^{\alpha}}
$$

and $\alpha=1.1$ provide a very good description of our experimental data. The inverse scaling factor $\tau_{\mathrm{c}}^{-1}$ is plotted as a function of temperature in the inset of Fig. 3(a). It

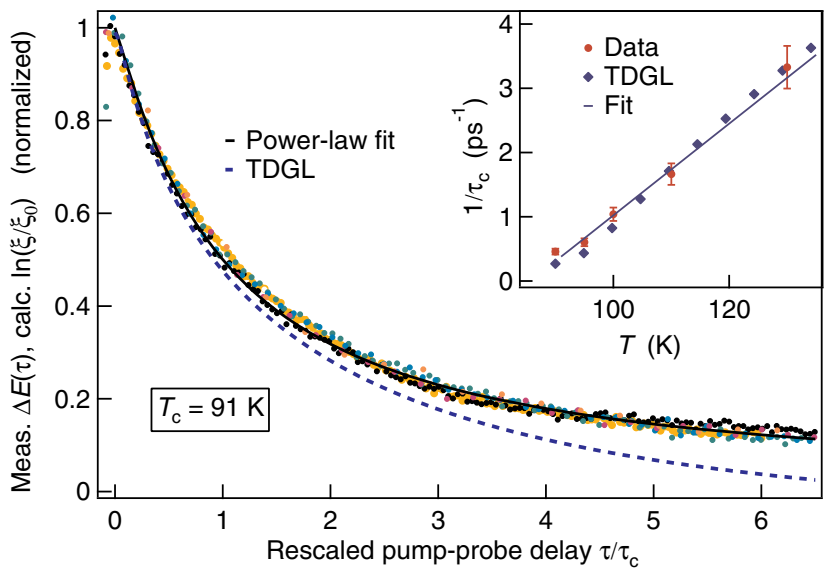

FIG. 3 (color online). Scaling behavior of ultrafast relaxation in the critical region between $T_{\mathrm{c}}$ and $T_{0}$ of optimally doped BSCCO $\left(T_{\mathrm{c}}=91 \mathrm{~K}\right)$. Measured pump-induced changes $\Delta E(\tau)$ in the transmitted electric field as a function of rescaled pump-probe delay $\tau / \tau_{\mathrm{c}}(T)$ at temperatures $T=90,95,100,110$, and $130 \mathrm{~K}$. Curves are also normalized to the maximum signal value. The solid black line is a power-law fit [see Eq. (1)] whereas the dashed line displays the logarithm of the GL correlation length as calculated using the TDGL model. The inset shows the scaling factor $\tau_{\mathrm{c}}^{-1}$ versus temperature as derived from the experiment (red circles) and TDGL theory (blue marks) as well as a linear fit (solid line).

approximately follows a linear relation $0.45 \mathrm{ps}^{-1}+$ $\left(T / T_{\mathrm{c}}-1\right) 6.5 \mathrm{ps}^{-1}$. The strong temperature dependence of $\tau_{\mathrm{c}}$ is consistent with a critical slowing down and increasing coherence length of superconducting fluctuations when approaching the transition temperature. We note that this is the classical regime of a second-order phase transition and is distinct from the quantum-critical fluctuations that have been suggested to explain the "strange metal" properties [28,35]. A critical scaling law of nonequilibrium fluctuations with $T \rightarrow T_{\mathrm{c}}$ is analyzed here for the first time. This finding goes substantially beyond equilibrium works [1-9] and reveals properties of the superconducting condensate far from equilibrium.

Theoretical model.-We now simulate our experimental findings using the two-dimensional, time-dependent Ginzburg-Landau (TDGL) model for the position (x)and time $(\tau)$-dependent order parameter $\psi(\boldsymbol{x}, \tau)[10,30]$. From a microscopic perspective, $\psi$ is the local pair potential leading to the single-particle gap. The TDGL model is justified for BSCCO due to the strong anisotropy of this layered compound. Temporal variations of the vector potential are neglected due to the large ratio between London penetration depth and coherence length [36,37]. In this coarse-grained description, the GL time $\tau_{\mathrm{GL}}$ is the characteristic response time of the order parameter $\psi(\boldsymbol{x}, \tau)$. The remaining degrees of freedom are assumed to evolve much faster than $\psi$ and merely remain as noise $\eta(\boldsymbol{x}, \tau)$ that affects the dynamics of $\psi$ as a source term. We note that, for our experimental conditions, the nonlinear term in the 
TDGL equation is very small, and the fluctuations can be considered, to a very good approximation, as a free classic field [30].

We identify the photoexcited state $\psi\left(\boldsymbol{x}, \tau^{*}\right)$ with a stationary solution of the TDGL equation at the elevated temperature $T+\Delta T$. The value $\Delta T=17 \mathrm{~K}$ is obtained by comparing the transient $\Delta E\left(\tau^{*}\right)$ of Fig. 2(b) with the equilibrium $\Delta E_{0}(T)$ of Fig. 1(c). In contrast, the fast degrees of freedom summarized in $\eta$ are assumed to return to their equilibrium configuration characterized by temperature $T$ directly after excitation. This sudden-quench hypothesis is motivated by the observation that uncondensed electrons in the normal phase transfer the pumpdeposited excess heat to the lattice within $\sim 100 \mathrm{fs}$ [26]. We model $\eta$ by white noise with the correlation function $\left\langle\eta(\boldsymbol{x}, \tau) \eta\left(\boldsymbol{x}^{\prime}, \tau^{\prime}\right)\right\rangle=2 S k_{\mathrm{B}} T \delta\left(\boldsymbol{x}-\boldsymbol{x}^{\prime}\right) \delta\left(\tau-\tau^{\prime}\right)$ where $S$ is the area of the sample film. Finally, for the GL time, we assume $\tau_{\mathrm{GL}}=\left(T / T_{\mathrm{c}}-1\right)^{-1} 0.25 \mathrm{ps}$, consistent with the estimate $\tau_{\mathrm{GL}}=48 \pi \sigma_{\mathrm{dc}} \lambda^{2} / c^{2}$ based on temperaturedependent measurements of penetration depth $\lambda$ and normal-state dc conductivity $\sigma_{\mathrm{dc}}[27,37,38]$. From our modeling, we calculate the superconducting coherence length as a function of time $\tau$ since optical excitation [30]. To enable comparison with the measured $\Delta E(\tau)$, we exploit the recent theoretical prediction [32] that the MIR conductivity of two-dimensional superconductors scales with $\ln \left(\xi / \xi_{0}\right)$.

Discussion.-As shown by Figs. 2(b), 2(c), and 3, the calculated $\ln \left(\xi / \xi_{0}\right)$ matches quite well the magnitude of the measured $\Delta E$ and the relaxation time for temperatures between $T_{\mathrm{c}}+5 \mathrm{~K}$ and $T_{0}$. Below $T_{\mathrm{c}}+5 \mathrm{~K}$, the TDGL overestimates $\tau_{\mathrm{c}}$ by $70 \%$. This discrepancy can be explained by an experimental crossover from the sudden-quench regime to an adiabatic regime occurring below $T_{\mathrm{c}}$ $[8,12,30]$. In addition, the experimental curves of Fig. 3 follow a power law, whereas the TDGL model predicts exponential relaxation. This discrepancy may originate from (a) an incomplete thermalization of the fast degrees of freedom, thereby violating the sudden-quench hypothesis, (b) the occurrence of coarsening phenomena related to extrinsic disorder, and (c) the signature of a conserved density coupled to the superconducting order parameter. In case (c), the TDGL (called model $A$ in the Halperin classification scheme) would not be adequate, whereas an effective model belonging to a different Halperin class could give better agreement with the experiment [13].

The limits of the TDGL description become more obvious in the critical dynamics of underdoped BSCCO. Figure 4(a) shows the photoinduced change $\Delta E\left(\tau^{*}\right)$ in the transmitted probe field measured for samples with $T_{\mathrm{c}}=68 \mathrm{~K}$. We identify the onset of superconducting fluctuations with the upturn of the signal at $T_{0}=100 \mathrm{~K}$. Note that $\Delta E\left(\tau^{*}\right)$ versus $T$ changes slope at the temperature $T^{*}=200 \mathrm{~K}$. This kink is likely due to the progressive reduction of the DOS known as "pseudogap." The value of
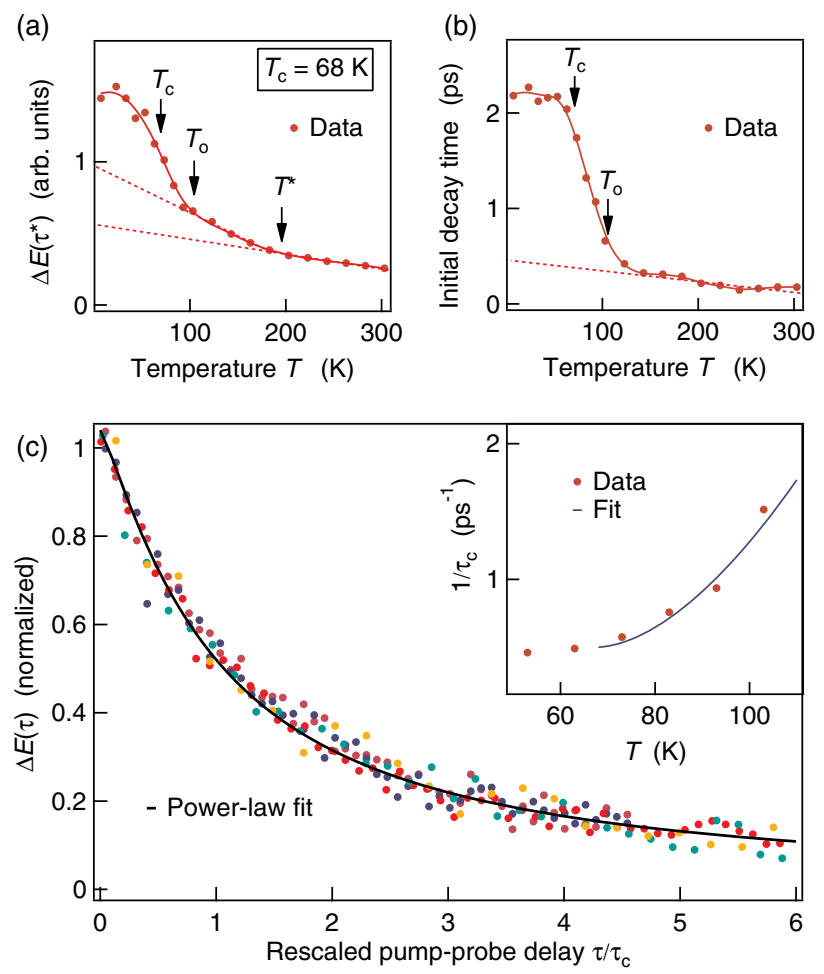

FIG. 4 (color online). Nonequilibrium data of underdoped $\operatorname{BSCCO}\left(T_{\mathrm{c}}=68 \mathrm{~K}\right)$ and their scaling behavior. (a) Magnitude of the pump-probe signal $\Delta E\left(\tau^{*}\right)$ and (b) its relaxation time $(\partial \ln \Delta E / \partial \tau)^{-1}$ right after sample excitation $\left(\tau^{*}=50 \mathrm{fs}\right)$ as a function of temperature (red circles). Transition temperature $T_{\mathrm{c}}=68 \mathrm{~K}$, onset of superconducting fluctuations $T_{0}=100 \mathrm{~K}$, and pseudogap temperature $T^{*}=200 \mathrm{~K}$ are indicated by arrows. (c) Measured pump-induced changes $\Delta E(\tau)$ of the transmitted electric field as a function of rescaled pump-probe delay $\tau / \tau_{\mathrm{c}}(T)$ at temperatures $T=68,78,88,98,108$, and $130 \mathrm{~K}$. Curves are also normalized to the maximum signal value. The solid black line is a power-law fit [see Eq. (1)]. The inset shows the scaling factor $\tau_{\mathrm{c}}^{-1}$ versus temperature as derived from the experiment (red circles) and a fit (solid line, see text).

$T^{*}$ found here compares well to other experiments on cuprates with similar doping levels [39]. As shown by Fig. 4(b), the initial instantaneous decay time $(\partial \ln \Delta E / \partial \tau)^{-1}$ displays a sudden upturn at the onset temperature $T_{0}=100 \mathrm{~K}$, whereas it is insensitive to the crossover at $T^{*}$. In contrast to the superconducting state, the pseudogap seems to lack any critical behavior. However, it is possible that correlations responsible for the pseudogap formation are strongly coupled to Cooper pairs and affect the dynamics of superconducting fluctuations near the critical point.

Figure 4(c) shows the relaxation dynamics of superconducting fluctuations in underdoped BSCCO. Strikingly, as with optimally doped BSCCO, all rescaled curves in the critical region between $T_{\mathrm{c}}$ and $T_{0}$ are found to obey the power law of Eq. (1). The value $\alpha=1.2$ provides the best fit to the experimental data and is very similar to the $\alpha=1.1$ found for optimally doped BSCCO (Fig. 3). The 
experimental evidence that the power law depends on the doping level only weakly is another hint of universality under far-from-equilibrium conditions. In contrast to optimally doped BSCCO (inset of Fig. 3), the temperature dependence of $\tau_{\mathrm{c}}^{-1}$ is not linear, but approximately follows the function $0.55 \mathrm{ps}^{-1}+\left(T / T_{\mathrm{c}}-1\right)^{\beta} 2.8 \mathrm{ps}^{-1}$ with $\beta=1.7$ [see inset of Fig. 4(c)]. This finding is in contrast to the predictions of the TDGL and calls for extended nonequilibrium models, perhaps including the coupling of the superconducting order parameter to other conserved densities [13].

In conclusion, we have observed universal behavior in the dynamics of superconducting fluctuations in hightemperature superconductors far from equilibrium. The temporal relaxation of the MIR conductivity follows the power law of Eq. (1), nearly independent of the doping level. We find that the critical temporal scaling factor behaves as $\left(T-T_{\mathrm{c}}\right)^{\beta}$ extending to temperatures of up to $1.5 T_{\mathrm{c}}$. The TDGL model reproduces some of the experimental results but suffers from inherent limits. Our alloptical approach is applicable to micrometer-sized samples and opens new perspectives in the field of critical systems far from equilibrium. Future work in this direction will potentially identify the symmetry of a coarse-grained model describing high-temperature superconductors [13] and impose stringent constraints to microscopic theories.

We acknowledge Elio Tosatti for enlightening discussions on critical phenomena. This work is supported by the research program "Investissements d'Avenir" LabEx PALM (Grant No. ANR-10-320 LABX-0039-PALM) and the German Research Foundation (Grant No. KA 3305/3-1).

[1] F. Rullier-Albenque, H. Alloul, and G. Rikken, Phys. Rev. B 84, 014522 (2011).

[2] J. Corson, R. Mallozzi, J. Orenstein, J. N. Eckstein, and I. Bozovic, Nature (London) 398, 221 (1999).

[3] L. S. Bilbro, R. Valdés Aguilar, G. Logvenov, O. Pelleg, I. Bozovic, and N. P. Armitage, Nat. Phys. 7, 298 (2011).

[4] N. Bergeal, J. Lesueur, M. Aprili, G. Faini, J. P. Contour, and B. Leridon, Nat. Phys. 6, 296 (2010).

[5] Z. Tesanovic, L. Xing, L. Bulaevskii, Q. Li, and M. Suenaga, Phys. Rev. Lett. 69, 3563 (1992).

[6] Y. Wang, L. Li, M. J. Naughton, G. D. Gu, S. Uchida, and N. P. Ong, Phys. Rev. Lett. 95, 247002 (2005).

[7] Y. Wang, L. Li, and N. P. Ong, Phys. Rev. B 73, 024510 (2006).

[8] M. Tinkham, Introduction to Superconductivity (McGrawHill, New York, 1996).

[9] A. Larkin and A. Valarmov, in Theory of Fluctuations in Superconductors (Oxford University Press, New York, 2005).

[10] M. Cyrot, Rep. Prog. Phys. 36, 103 (1973).

[11] L. P. Gorkov, Sov. Phys. JETP 9, 1364 (1959).
[12] A. Polkovnikov, K. Sengupta, A. Silva, and M. Vengalattore, Rev. Mod. Phys. 83, 863 (2011).

[13] P. C. Hohenberg and B. I. Halperin, Rev. Mod. Phys. 49, 435 (1977).

[14] G. Lamporesi, S. Donadello, S. Serafini, F. Dalfovo, and G. Ferrari, Nat. Phys. 9, 656 (2013).

[15] J. Demsar, B. Podobnik, V. V. Kabanov, Th. Wolf, and D. Mihailovic, Phys. Rev. Lett. 82, 4918 (1999).

[16] V. V. Kabanov, J. Demsar, and D. Mihailovic, Phys. Rev. Lett. 95, 147002 (2005).

[17] C. Giannetti et al., Nat. Commun. 2, 353 (2011).

[18] G. P. Segre, N. Gedik, J. Orenstein, D. A. Bonn, R. Liang, and W. N. Hardy, Phys. Rev. Lett. 88, 137001 (2002).

[19] I. Madan, T. Kurosawa, Y. Toda, M. Oda, T. Mertelj, P. Kusar, and D. Mihailovic, Sci. Rep. 4, 5656 (2014).

[20] R. A. Kaindl et al., Science 287, 470 (2000).

[21] A. Pashkin et al., Phys. Rev. Lett. 105, 067001 (2010).

[22] R. D. Averitt, G. Rodriguez, A. I. Lobad, J. L. W. Siders, S. A. Trugman, and A. J. Taylor, Phys. Rev. B 63, 140502 (2001).

[23] R. A. Kaindl, M. A. Carnahan, D. S. Chemla, S. Oh, and J. N. Eckstein, Phys. Rev. B 72, 060510 (2005).

[24] W. Hu, S. Kaiser, D. Nicoletti, C. R. Hunt, I. Gierz, M. C. Hoffmann, M. Le Tacon, T. Loew, B. Keimer, and A. Cavalleri, Nat. Mater. 13, 705 (2014).

[25] M. A. Carnahan, R. A. Kaindl, J. Orenstein, D. S. Chemla, S. Oh, and J. N. Eckstein, Physica C (Amsterdam) 408-410, 729 (2004).

[26] L. Perfetti, P. A. Loukakos, M. Lisowski, U. Bovensiepen, H. Eisaki, and M. Wolf, Phys. Rev. Lett. 99, 197001 (2007).

[27] M. A. Quijada, D. B. Tanner, R. J. Kelley, M. Onellion, H. Berger, and G. Margaritondo, Phys. Rev. B 60, 14917 (1999)

[28] D. van der Marel, H. J. A. Molegraaf, J. Zaanen, Z. Nussinov, F. Carbone, A. Damascelli, H. Eisaki, M. Greven, P. H. Kes, and M. Li, Nature (London) 425, 271 (2003).

[29] D. N. Basov and T. Timusk, Rev. Mod. Phys. 77, 721 (2005).

[30] See Supplemental Material at http://link.aps.org/ supplemental/10.1103/PhysRevLett.114.067003 for details on data analysis and modeling.

[31] S. G. Sharapov and J. P. Carbotte, Phys. Rev. B 72, 134506 (2005).

[32] F. Federici and A. A. Varlamov, Phys. Rev. B 55, 6070 (1997).

[33] C. L. Smallwood, J. P. Hinton, C. Jozwiak, W. Zhang, J. D. Koralek, H. Eisaki, D.-H. Lee, J. Orenstein, and A. Lanzara, Science 336, 1137 (2012).

[34] A. Bray, Adv. Phys. 51, 481 (2002).

[35] C. M. Varma, P. B. Littlewood, S. Schmitt-Rink, E. Abrahams, and A. E. Ruckenstein, Phys. Rev. Lett. 63, 1996 (1989).

[36] Q. Du and P. Gray, SIAM J. Appl. Math. 56, 1060 (1996).

[37] J. Mosqueira, E. G. Miramontes, C. Torron, J. A. Campa, I. Rasines, and F. Vidal, Phys. Rev. B 53, 15272 (1996).

[38] S. Martin, A. T. Fiory, R. M. Fleming, G. P. Espinosa, and A. S. Cooper, Phys. Rev. Lett. 62, 677 (1989).

[39] M. R. Norman, D. Pines, and C. Kallin, Adv. Phys. 54, 715 (2005). 